\title{
Review of: "Forecasting renewable energy for environmental resilience through computational intelligence"
}

\author{
Mustafa Jaihuni
}

Potential competing interests: The author(s) declared that no potential competing interests exist.

Points to be addressed:

1. In the introduction part, the author has pointed out contributions of the paper. But the listed points are more like an explanation of the methodology used in this work. Specific and unique contributions of this work are needed to be mentioned i.e. what is new in this study; what new findings or methods are introduced to the existing literature.

2. What does NREL stand for? Please mention it in the text where it is mentioned for the first time.

3. Please give a reference for the below claim in the Literature Review part: "Most recent researches have been focused on hybrid techniques to take advantage of integrated methods."

4. The Literature Review part is very long and can be shortened.

5. In Tables 2-3-4, how were the hyper-parameters selected? Explain in details.

6. In the methodology section, there should be a part indicating the ways you would evaluate performances of the models? Additionally, indicate why you used MAE, RMSE over others.

7. Please indicate the formula used for $R^{2}$ correlation calculations. As the $R^{2}$ values near 1 shows how fit the predicted values are to the actual ones.

8. How did you treat overfitting issues in the CNN-LSTM models? As shown in figures 5-7, the predicted values are quite close to the actual values. 